A

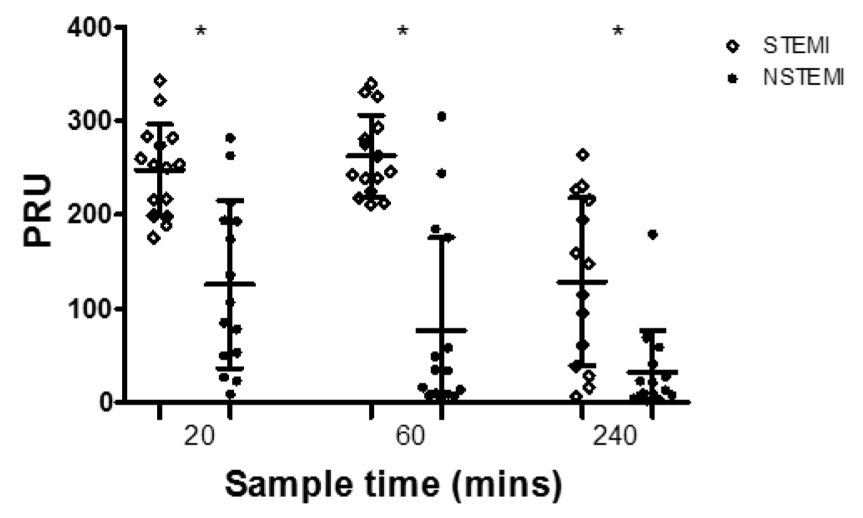

B

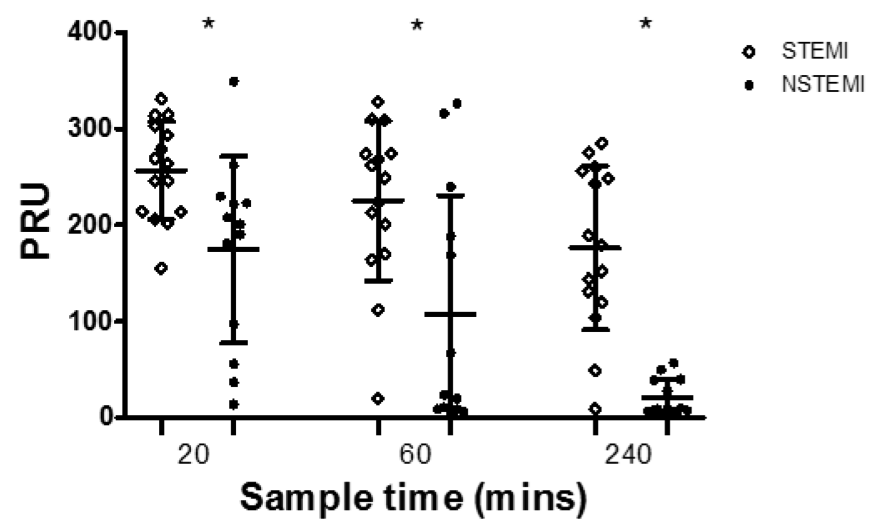

Abstract 109 Figure 1 The degree of inhibition of platelet reactivity (expressed as PRU) over time following the administration of prasugrel (A) and ticagrelor (B) in STEMI and NSTEMI patients. PRU = P2Y12 reactivity units

difference in the effect of ticagrelor vs prasugrel over time in either STEMI or NSTEMI/UA.

Conclusion Prasugrel and ticagrelor in the context of STEMI do not provide adequate P2Y12 inhibition at reperfusion and the first hour post loading when compared to patients with NSTEMI/UA.

\begin{tabular}{llll} 
Abstract & 09 Table & \multicolumn{3}{l}{ Baseline characteristics } \\
\hline Characteristic & STEMI (N=30) & NSTEMI (N=28) & P-value \\
\hline Age (yrs) & $59.94 \pm 12.68$ & $61.61 \pm 11$ & 0.595 \\
Female & 7 & 3 & 0.301 \\
Diabetes Mellitus & 6 & 12 & 0.089 \\
Hypertension & 14 & 13 & 1 \\
Current Smoker & 7 & 4 & 0.508 \\
Ex-Smoker & 12 & 11 & 1 \\
Hyperlipidaemia & 8 & 16 & 0.032 \\
Familial History of CAD & 17 & 15 & 1 \\
\hline CAD = Coronary Artery Disease & &
\end{tabular}

110 THE PROTHROMBOTIC RISK OF PATIENTS WITH TYPE 2 DIABETES IN STABLE AND UNSTABLE CORONARY ARTERY DISEASE

${ }^{1}$ Martin Berger*, 'Katharina Schütt, 'Katharina Lysaja, ${ }^{1}$ Michael Lehrke, ${ }^{2}$ Nikolaus Marx. ${ }^{1}$ UKAachen - Department of Internal Medicine 1; ${ }^{2}$ UKAachen - Department of Internal Medicine l; *Presenting Author

\subsection{6/heartjnl-2016-309890.110}

Aims/hypothesis Clot properties are altered in acute coronary syndromes (ACS). However, data on clot properties and the impact of concomitant disease and medication in patients with diabetes in ACS are incomplete. Therefore, the present study investigates clot parameters in stable and unstable coronary artery disease (SCAD and UCAD respectively).

Methods Hundred-eighty patients were included in a consecutive manner based on their diabetes and CAD status between March 2012 and December 2014. Clot properties were determined by a turbidimetric assay in 90 controls (noCAD N=39; SCAD $\mathrm{N}=29$; , UCAD $\mathrm{N}=22$ ) and 90 patients with diabetes (noCAD N=21; SCAD N=41; UCAD N=28).

Results Clot structure was not affected by CAD status. However, clot lysis time was significantly increased in UCAD compared to SCAD and absence of CAD in control patients $(1414 \pm 703,915 \pm 461$ and $1069 \pm 414$ respectively; $\mathrm{p}=$ 0.003). In contrast, in patients with DM clot lysis time did not differ between UCAD, SCAD and absence of CAD (1260 $\pm 649,1304 \pm 658$ and $1318 \pm 675$ respectively; $\mathrm{p}=$ 0.947). Interestingly, clot lysis time in diabetes patients without CAD was comparable to UCAD control patients $(\mathrm{p}=$ 0.654). In an adjusted multiple regression model clot lysis time was significantly predicted by PAI-1 $(\mathrm{p}=0.023)$, CRP $(\mathrm{p}$ $=0.042$ ) and presence of UCAD (NSTEMI $\mathrm{p}=0.010$, STEMI $\mathrm{p}=0.002)$ in control patients. Strikingly, in diabetes patients solely PAI-1 $(\mathrm{p}=0.004)$ predicted clot lysis time.

Conclusions Unstable coronary artery disease leads to an increase in clot lysis time in control patients. In contrast, clot lysis time in patients with diabetes is not affected by UCAD. Strikingly, clot lysis time in diabetes patients without CAD is comparable to control patients with UCAD indicating their increased prothrombotic risk already present in a stable situation.

\section{THE DEGREE AND TIME COURSE OF PLATELET INHIBITION FOLLOWING THE ADMINISTRATION OF ORAL ANTIPLATELET AGENTS IN PATIENTS PRESENTING WITH ST ELEVATION MI (STEMI)}

${ }^{1}$ Nazish Khan*, ${ }^{2}$ Amoah Vincent, ${ }^{2}$ Andrew Smallwood, ${ }^{2}$ Ben Wrigley, ${ }^{3}$ Joe Martins, ${ }^{2}$ Saib Khogali, ${ }^{2}$ Munir Shahzad, ${ }^{4}$ Nevill Alan, ${ }^{2}$ James Cotton. ${ }^{1}$ The Royal Wolverhampton Hospitals NHS Trust; ${ }^{2}$ The Royal Wolvehampton Hospitals NHS Trust; ${ }^{3}$ The Dudley Group of Hospitals; ${ }^{4}$ University of Wolverhampton; *Presenting Author

\subsection{6/heartjnl-2016-309890.111}

Introduction Oral P2Y12 inhibitors have proven clinical efficacy in a variety of cardiological settings. The degree and time course of platelet inhibition using common P2Y12 inhibitors during the acute phase of a myocardial infarction is an under explored area. We aimed to determine the effect of clopidogrel, prasugrel and ticagrelor in the first four hours following loading in patients admitted with STEMI undergoing primary percutaneous coronary intervention (PPCI). 